OPEN ACCESS

Edited by:

Albert Giralt,

University of Barcelona, Spain

Reviewed by:

Francesc Miro

August Pi i Sunyer Biomedical Research Institute (IDIBAPS), Spain

Seong-Woon Yu,

Daegu Gyeongbuk Institute of Science and Technology (DGIST),

South Korea

*Correspondence:

Qiang Liu

qliu.asu@gmail.com

Hongshan Ma

dtsyy2009@126.com

Minshu Li

minshuli2012@163.com

Specialty section:

This article was submitted to Non-Neuronal Cells, a section of the journal Frontiers in Cellular Neuroscience

Received: 28 February 2020 Accepted: 11 May 2020 Published: 08 June 2020

Citation:

Li X, Cheng X, Wang X, Liu Q, Ma H and Li M (2020) Dyslipidemic Diet Induces Mobilization of Peripheral Neutrophils and Monocytes That Exacerbate Hemorrhagic Brain Injury and Neuroinflammation. Front. Cell. Neurosci. 14:154 doi: 10.3389/fncel.2020.00154

\section{Dyslipidemic Diet Induces Mobilization of Peripheral Neutrophils and Monocytes That Exacerbate Hemorrhagic Brain Injury and Neuroinflammation}

\author{
Xiuping $\mathrm{Li}^{1,2}$, Xiaojing Cheng ${ }^{1,2}$, Xuejiao Wang ${ }^{3}$, Qiang Liu ${ }^{1,2 *}$, Hongshan Ma ${ }^{3 *}$ \\ and Minshu $L i^{1,2,4,5,6 *}$
}

\begin{abstract}
${ }^{1}$ Department of Neurology, Tianjin Neurological Institute, Tianjin Medical University General Hospital, Tianjin, China, ${ }^{2}$ Key Laboratory of Post-neurotrauma Neuro-repair and Regeneration in Central Nervous System, Tianjin Neurological Institute, Ministry of Education, Tianjin, China, ${ }^{3}$ Center for Neurological Diseases, The Third People's Hospital of Datong, Shanxi, China, ${ }^{4}$ China National Clinical Research Center for Neurological Diseases, Beijing Tiantan Hospital, Capital Medical University, Beijing, China, ${ }^{5}$ Advanced Innovation Center for Human Brain Protection, Capital Medical University, Beijing, China, ${ }^{6}$ Beijing Key Laboratory of Translational Medicine for Cerebrovascular Disease, Beijing, China
\end{abstract}

Background: Non-alcoholic fatty liver disease (NAFLD) is a common liver condition characterized by a significant accumulation of lipids in the liver without excessive alcohol consumption. Accumulating evidence suggests a significantly increased risk of intracerebral hemorrhage $(\mathrm{ICH})$ in NAFLD patients. However, it remains poorly understood whether and how NAFLD affects the outcome of hemorrhagic brain injury. Here, we examined the effects of diet-induce NAFLD on ICH injury and neuroinflammation in mice.

Methods: NAFLD was induced in C57BL/6 mice by feeding with a methionine-choline deficient (MCD) diet for 4 weeks. Collagenase and autologous blood models were used to evaluate the effects of NAFLD on ICH injury and neuroinflammation.

Results: MCD diet for 4 weeks induces NAFLD and hyperlipidemia in mice. Mice receiving the MCD diet have aggravated neurological deficits and brain edema after $\mathrm{ICH}$. The augmentation of $\mathrm{ICH}$ injury was accompanied by brain infiltration of neutrophils and monocytes and increased production of pro-inflammatory factors. Before ICH, MCD diet-induced mobilization of neutrophils and monocytes in the periphery. Notably, the detrimental effects of NAFLD on ICH injury was ablated in mice receiving antibody depletion of neutrophils and monocytes.

Conclusions: These results suggest that NAFLD exacerbates neuroinflammation and $\mathrm{ICH}$ injury.

Keywords: intracerebral hemorrhage, NAFLD, neuroinflammation, myeloid cells, immune response

\footnotetext{
Abbreviations: NAFLD, Non-alcoholic fatty liver disease; ICH, Intracerebral hemorrhage; MCD, Methionine cholinedeficient; mNSS, Modified neurological severity score; MMP-9, Matrix metalloproteinase-9; PCR, Polymerase chain reaction; MFI, Mean fluorescence intensity; BBB, Brain blood barrier; NASH, Nonalcoholic steatohepatitis.
} 


\section{INTRODUCTION}

Intracerebral hemorrhage (ICH) is a severe type of stroke without effective treatment (Aronowski and Zhao, 2011). Epidemiological studies have identified that environmental and dietary factors are related to the incidence and outcome after $\mathrm{ICH}$. Among factors that increase the risk of $\mathrm{ICH}$, non-alcoholic fatty liver disease (NAFLD) is a common liver disease that affects up to $30 \%$ of the general population (Tsochatzis and Newsome, 2018). NAFLD is characterized by excessive deposition of lipids in liver parenchyma independent of heavy alcohol consumption (Kim et al., 2011; Younossi et al., 2018; Khoury et al., 2019). Accompanying the accumulation of hepatic fat, patients usually display insulin resistance, dyslipidemia, and escalated systemic inflammation (Gluchowski et al., 2017; Fiorucci et al., 2018). However, it remains elusive whether and how NAFLD affects ICH outcome.

Emerging evidence has demonstrated inflammation as a key element that contributes to secondary brain injury after ICH (Zhou et al., 2014). Following the ictus of ICH, blood components including leukocytes surge into the brain and activate resident glia and recruit immune cells such as neutrophils and monocytes (Mracsko et al., 2014; Sheth and Rosand, 2014; Zhang Z. et al., 2017). These inflammatory events accelerate the development of perihematomal edema and neurological deterioration (Haukeland et al., 2006; Farrell et al., 2012; Gao and Tsukamoto, 2016). Clinical studies have indicated that patients with NAFLD are characterized by an augmentation of systemic inflammation characterized by increased mobilization of circulating inflammatory cells, suggesting that NAFLD possibly induces mobilization of peripheral immune cells. Yet the influences of such mobilization on the pathological responses to ICH remain unknown. To address this question, we induced NAFLD by feeding mice with a methionine-choline deficient (MCD) diet and investigated the effects of NAFLD on hemorrhagic injury and neuroinflammation using two ICH models.

\section{MATERIALS AND METHODS}

\section{Animals}

Experimental protocols were approved by the Institutional Animal Care and Use Committees of Tianjin Medical University General Hospital. All experiments were designed, conducted, and reported according to the ARRIVE (Animal Research: reporting of in vivo Experiments) guidelines. Male C57BL/6 mice (12 weeks old) were purchased from the Vital River Corporation (Beijing, China). Mice were housed in pathogen-free conditions with free access to food and water. All Surgeries on mice were performed under anesthesia. All mice were randomly assigned to each experiment.

\section{Induction of ICH in Mice}

As we previously reported, ICH was induced in mice by injection of autologous blood or bacterial collagenase (Li et al., 2017a,b; Ren et al., 2018). First, mice were anesthetized with an intraperitoneal injection of ketamine $(100 \mathrm{mg} / \mathrm{kg})$ and xylazine
$(10 \mathrm{mg} / \mathrm{kg})$. After placing the mice on a stereotactic frame, a 1-mm burr hole was drilled on the right side of the skull $(2.3 \mathrm{~mm}$ lateral to the midline, $0.5 \mathrm{~mm}$ anterior to the bregma). For the collagenase ICH model, $0.0375 \mathrm{U}$ bacterial collagenase (Type IV-S, Sigma, St. Louis, MO, USA) dissolved in $0.5 \mu \mathrm{l}$ saline was infused at the caudate nucleus $(3.7 \mathrm{~mm}$ depth beneath the skull) through an infusion pump (Kd Scientific Inc., Holliston, MA, USA) at a rate of $0.5 \mu \mathrm{l} / \mathrm{min}$. In some experiments, we induced the mouse ICH model by infusion of autologous blood using a double-injection method. Whole blood $(30 \mu \mathrm{l})$ was withdrawn from the angular vein and then infused into the brain as previously described (Sun et al., 2016). $5 \mu$ l of blood was first injected to generate a clot at a depth of $3 \mathrm{~mm}$ beneath the hole. Then the needle was moved to a depth of $3.5 \mathrm{~mm}$ to inject the remaining $25 \mu \mathrm{l}$ of blood at the rate of $1 \mu \mathrm{l} / \mathrm{min}$. After surgery, animals were placed in cages and provided with free access to food and water.

\section{Study Design and Drug Administration}

A total of 207 male C57BL/6 mice were used in this study. Mice were randomly assigned to each group according to the type of chow they were given. A methionine-choline deficient (MCD) diet (H10401, Beijing HFK Bioscience Company Limited) was fed for 4 weeks to produce the animal model of NAFLD before $\mathrm{ICH}$ induction. Mice fed the normal diet were used as controls. This administration persisted at the end of the experiment (Larter and Yeh, 2008; Zhang et al., 2014). The mouse ICH model was induced by infusion of collagenase or autologous blood at day 0 . A series of assessments were performed at indicated time points after ICH. For the Gr-1 $1^{+}$cell depletion experiment, an anti-mouse Gr-1 monoclonal antibody (MAbRB6-8C5; BioXcell, West Lebanon, NH, USA) was delivered by intraperitoneal injection 1 day before and 1 day after $\mathrm{ICH}$ induction at a dose of $250 \mu \mathrm{g}$ per mouse (Condamine et al., 2014; Wang et al., 2015). More than $90 \%$ of $\mathrm{Gr}-1^{+}$cells were depleted in mice receiving an anti-mouse Gr-1 monoclonal antibody. All data were analyzed by independent investigators blinded to the group assignment.

\section{Behavioral Assessment}

Behavioral assessment was performed at indicated time points after ICH surgery to assess the motor, sensory, reflex, and balance functions. The modified Neurological Severity Score (mNSS), corner turning test, together with the foot-fault test were conducted as previously described (Clarkson et al., 2010; Klebe et al., 2017; Ren et al., 2018). The range of scores for mNSS is from 0 to 18 and mice were given 1 point for the inability to fulfill a task. The corner turning test was used to assess sensorimotor and postural asymmetries. Briefly, each mouse freely proceeds into a corner with an angle of 30 degrees and then turns right or left to exit. This task was repeated 10 times with at least $30 \mathrm{~s}$ intervals between trials. The percentage of right turns was calculated and recorded. For the foot-fault test, mice were placed on a grid device measuring $32 \mathrm{~cm} \times 20 \mathrm{~cm} \times 50 \mathrm{~cm}$ (length $\times$ width $\times$ height) with $12-\mathrm{mm}$ mesh and allowed to roam freely for $5 \mathrm{~min}$. A foot fault was defined as the mouse dropping its limb into the grid hole or resting with the grid at the 
wrist level. The percentage of foot faults was calculated according to the formula: foot faults/(foot fault + nonfoot fault steps $) \times 100$.

\section{Brain Water Content Measurement}

Brain water content was assessed on day 3 after ICH induction, as previously described (Han et al., 2017). Briefly, mice brains were removed without perfusion and dissected into three parts: the ipsilateral, the contralateral, and cerebellum. The collected tissues were weighed immediately to obtain wet weights and then dried for $24 \mathrm{~h}$ at $100^{\circ} \mathrm{C}$ to get dry weights. The percentage of water content was calculated using the formula: (wet weight-dry weight)/wet weight $\times 100 \%$.

\section{Flow Cytometry}

At days 3 after $\mathrm{ICH}$, the brain, spleen, and peripheral blood of mice were obtained for flow cytometry analysis to detect the counts of immune cells and cytokine production. Single-cell suspensions were prepared as previously described (Jin et al., 2016; Liu et al., 2016; Yang et al., 2019). Briefly, mice were perfused with cold PBS, thereafter mouse brain tissues were harvested and digested with collagenase IV to form a single cell suspension. After the myelin was removed using a 30\% Percoll solution (GE Healthcare Bio-Science AB, Uppsala, Sweden), cell pellets were harvested on the bottom of the tube and suspended in $1 \%$ BSA solution for staining. To harvest splenic cells, the spleen tissues were removed and pressed through a $70 \mu \mathrm{m}$ nylon cell strainer in PBS. After lysis of red blood cells, cell pellets were prepared for staining. Blood samples were collected from mice by eyeball extirpating and were subjected to red blood cell lysis. Cell pellets were harvested and collected for staining. All antibodies used were purchased from Biolegend (San Diego, CA, USA) unless otherwise indicated. The following antibodies were used in this study: CD45 (30-F11), CD11b (M1/70), CD3 (145-2C11), CD4 (GK1.4), CD8 (53-6.72), CD19 (1D3), Ly6C (HK1.4), Ly6G (1A8). Rabbit anti-MMP-9 (ab38898) antibody or Rabbit IgG, polyclonal - Isotype Control (ab37415) was purchased from Abcam (Cambridge, MA, USA), and Alexa Fluor ${ }^{\circledR} 488$ conjugated goat anti-rabbit IgG $(\mathrm{H}+\mathrm{L})$ was used as the secondary antibody (ab150077, Abcam). Meanwhile, the isotype controls were stained, respectively. The staining process followed the protocol in the manual and flow cytometry data were obtained using a FACS Aria III (BD Bioscience, San Jose, CA, USA). The data were analyzed by Flow Jo version 7.6.1 (flowjo.com). For the procedure for absolute counting of cells using flow cytometry, we adopted two methods to count interested cell populations in flow cytometry. One is that we recorded the volume of cells that have passed through the flow. The gate on the interested population and work out the concentration of cells (events recorded/volume recorded). With that concentration and a defined volume of cells to begin with we can work out the absolute cell number from the original volume (= concentration $\times$ volume). The other one is that we counted the total number of single cells harvested from the brain or spleen by trypan blue method. After staining, then we run and gate our interested cell population using flow cytometry. The formula we calculated cell number is the total number multiplied by our interested cell population step by step.

\section{Real-Time Polymerase Chain Reaction}

Three days after ICH induction, total RNA was extracted from the ipsilateral hemisphere of brain tissue using Trizol reagent (Invitrogen, Carlsbad, CA, USA) according to the manufacturer's instructions. The concentration of RNA was measured by ultraviolet spectrophotometry at 260/280 $\mathrm{nm}$. Then total RNA was reverse transcribed into cDNA utilizing the PrimeScript ${ }^{\mathrm{TM}}$ RT reagent Kit (TaKaRa, Shiga, Japan). All procedures were conducted as instructed. SYBR Green PCR Master Mix (Roche, Indianapolis, IN, USA) was used to amplify the gene sequences on the Opticon 2 Real-Time PCR Detection System (BioRad, Hercules, CA, USA). The primers used in this experiment are listed in Supplementary Table S1. GAPDH served as a reference gene and mRNA expression was calculated as fold changes using the $2^{-\Delta \Delta C t}$ method. The primer sequences used for RT-PCR analysis in this study were listed in Supplementary Table S1.

\section{Assessment of BBB Permeability}

On day 3 after $\mathrm{ICH}, \mathrm{BBB}$ permeability was measured by quantification of Evans Blue (Sigma, St. Louis, MO, USA) extravasation, as previously described (Zhang Y. et al., 2017; Ren et al., 2018). Evans Blue (2\% in saline, $4 \mathrm{ml} / \mathrm{kg}$ ) was injected through the caudal vein $2 \mathrm{~h}$ before the brain was collected. the mice were anesthetized with $10 \%$ chloral hydrate and perfused with PBS. After decapitation, the brains were removed and slabbed. Tissues were then photographed for qualitative analysis. For quantitative analysis, the brain tissues were removed, weighed, and placed in dimethylformamide. After homogenization, the mixture was incubated for $72 \mathrm{~h}$ in a $60^{\circ} \mathrm{C}$ water bath and centrifuged at $1,500 \mathrm{~g}$ for $10 \mathrm{~min}$. The absorbance value of supernatant was measured by the fluorescence spectrophotometer. The tissue content of Evans Blue was quantified from a linear standard curve.

\section{Hematoxylin \& Eosin (H\&E) Stain, Oil Red O Stain}

After mice received an MCD diet or control diet for 4 weeks, liver tissues were harvested and fixed using $4 \%$ paraformaldehyde. Then, $8 \mu \mathrm{m}$-thick sections were prepared with a microtome (Leica Biosystems, Nussloch, Germany). After dewaxing and rehydrating, the tissue sections were stained with $\mathrm{H} \& \mathrm{E}$ using a commercial kit (Solarbio, Beijing, China). Microscopic images were obtained using a microscope (Olympus, Tokyo, Japan). For Oil Red O stain, the fixed liver tissues were embedded with OCT and sliced into $8 \mu \mathrm{m}$-thick sections. After washing in distilled water, slices were soaked in $60 \%$ isopropyl alcohol for 20-30 s. Then, the slices were placed in a dyeing tank filled with a modified oil red O staining solution (G1261, Solarbio Life Sciences) and stained for 10-15 min in the dark. After separating and washing, the slices were counterstained with hematoxylin solution for 1-2 min to reveal the nuclei. Finally, slices were mounted with the glycerin gelatin for observation.

\section{Assessment of Triglyceride (TG)}

Lipids in the serum and liver were measured according to the manual of the triglyceride assay kit (A110-2-1, 
Nanjing Jiancheng Bioengineering Institute, Nanjing, China). Triglyceride (TG) from serum and liver samples was estimated by enzymatic colorimetric GPO-PAP method. The whole blood was centrifuged to separate serum. For the liver tissue, the supernatant was centrifuged and collected after homogenization. The sample was mixed with the working solution and incubated for $10 \mathrm{~min}$ at $37^{\circ} \mathrm{C}$. The absorbance was measured at $510 \mathrm{~nm}$. The contents of triglyceride were measured by calculating the absorbance value of the standard and the sample.

\section{Coagulation Time and Bleeding Time Measurement}

To assess the coagulation time of mice, blood was acquired from the angular vein using glass capillary after anesthesia. Then the tube filled with blood was put on a flat top. Equal length of the capillary tube was snapped every $30 \mathrm{~s}$ until a fibrin thread formed. As for the bleeding time of mice, we used a scalpel blade to transect the tail starting from $2 \mathrm{~mm}$ off the tip. The tail was immersed in a clear tube filled with saline at $37^{\circ} \mathrm{C}$. The bleeding time was defined as time to cessation of bleeding. The maximum observing time was $20 \mathrm{~min}$. If there was no bleeding within $30 \mathrm{~s}$, it was considered as stopped.

\section{Statistical Analysis}

The sample size was determined by power analysis adopting a significance level of $\alpha=0.05$ with $80 \%$ power to assess significant differences. We performed the power analysis and sample size calculations using SAS 9.1 software (SAS Institute Inc., Cary, NC, USA). Data were analyzed by at least two investigators blinded to group assignment. All values are shown as mean $\pm \mathrm{SD}$. Statistical analyses were conducted using Graphpad 6.0 software. The unpaired 2-tailed Student's $t$-test was used for determining the significance of differences between the two groups and the survival index was analyzed by Log-rank (Mantel-Cox) test. $P<0.05$ is considered significant.

\section{RESULTS}

\section{NAFLD Exacerbates Brain Injury and Neurological Outcome After ICH}

To determine the impact of NAFLD on brain injury after $\mathrm{ICH}$, we examined neurological deficits and brain water content in $\mathrm{ICH}$ mice receiving an MCD diet or normal control diet. Before ICH induction, mice were fed with an MCD diet or control diet for four consecutive weeks (Figure 1A). After 4 weeks of MCD diet, dramatic lipid accumulation in the liver and hyperlipidemia were observed while coagulation time remained unaltered (Supplementary Figure S1). After ICH surgery, these mice continued to receive an MCD diet or control diet until the end of experiments (Figure 1A). We found that NAFLD mice receiving MCD diet had significantly increased

A
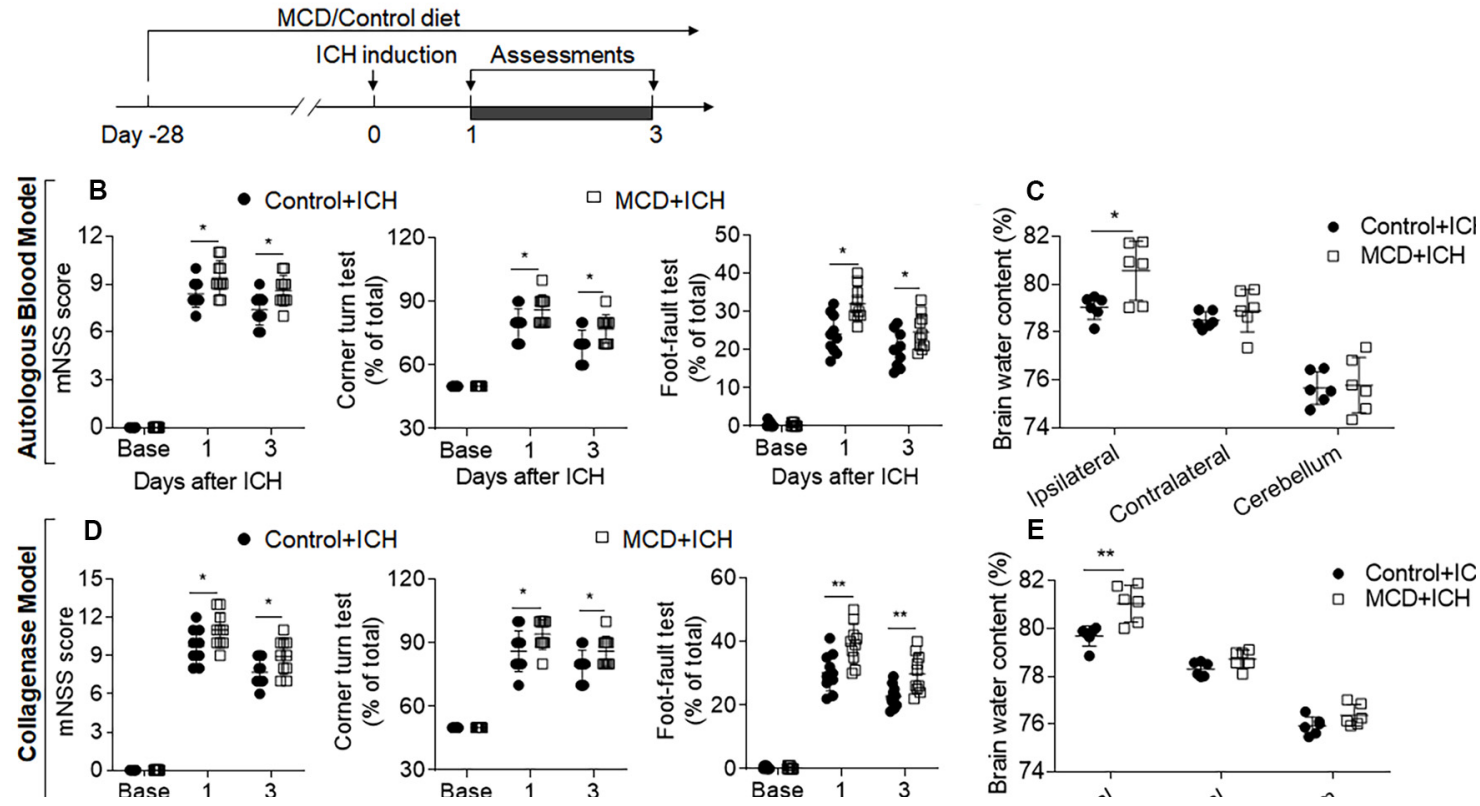

FIGURE 1 | Non-alcoholic fatty liver disease (NAFLD) aggravated neurological deficits and brain edema in intracerebral hemorrhage (ICH) mice. (A) Flow chart illustrates the individual diet administration and experimental design. Mice were fed the methionine-choline deficient (MCD) diet for 4 weeks to develop the NAFLD model, and this administration persisted to the end of the experiment. ICH was induced in C57BL/6 mice by injection of autologous blood or collagenase at day 0 . Neurological deficits and brain water content were measured at days 1 and 3. (B,D) The statistical data of mNSS, Corner turning test, and foot-fault test in ICH mice with control diet or MCD diet at baseline, days 1 and 3 after injection of autologous blood (B) or collagenase (D). $n=10$ mice per group. (C,E) The Brain water content in mice with the control or MCD diet in autologous blood model (C) or collagenase model (E) on day 3 after ICH. $n=6$ mice per group. Data are presented as mean $\pm \mathrm{SD}$. ${ }^{\star} P<0.05,{ }^{\star \star} P<0.01$. 

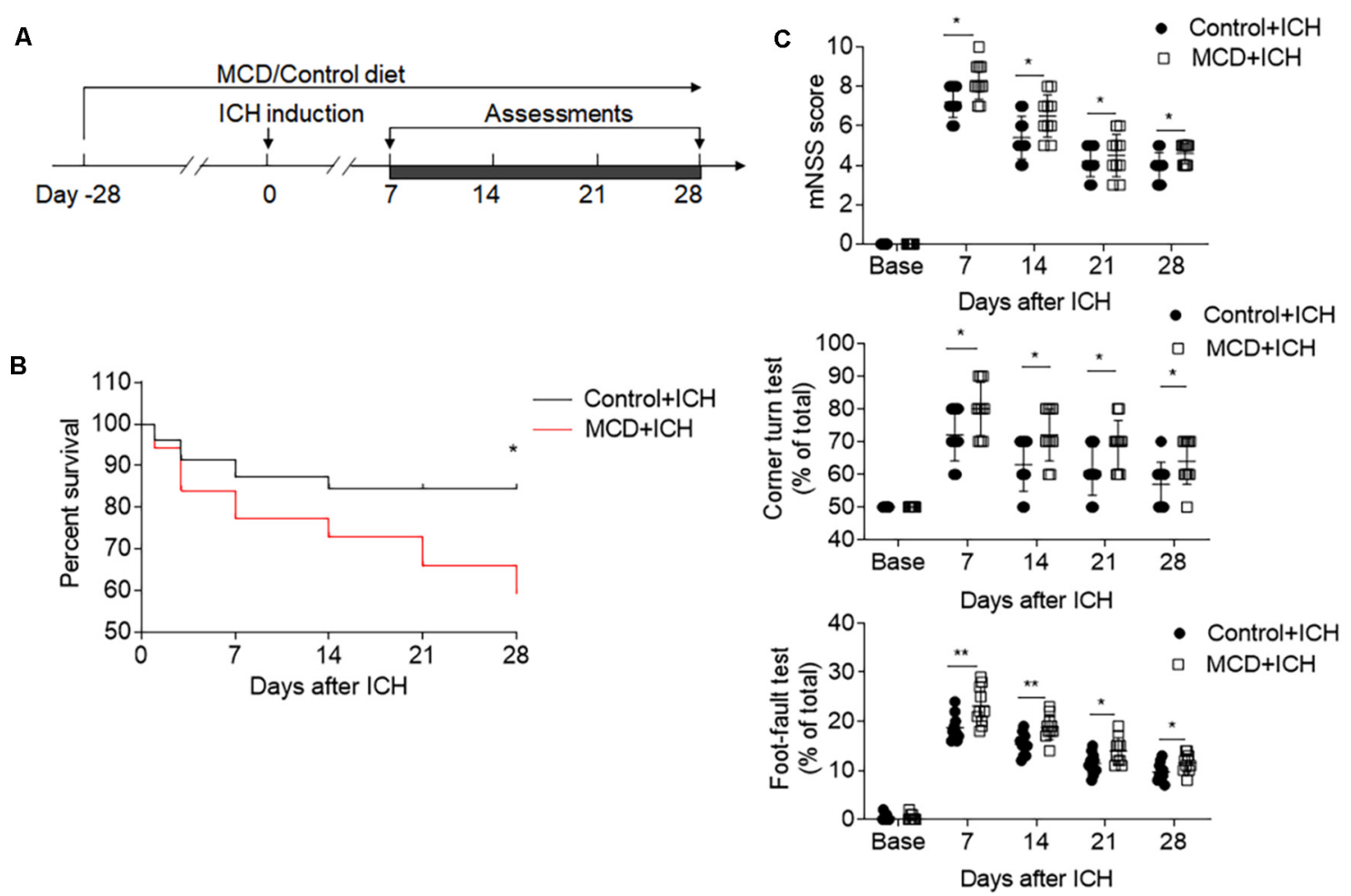

FIGURE 2 | NAFLD led to the worse neurological outcome and increased mortality after ICH. ICH was induced in mice by injection of collagenase. A battery of neurological tests was conducted to assess the motor, sensory, and balance functions in two groups of mice at days 7 , 14, 21, and 28 after ICH. (A) Flow chart illustrates the individual diet administration and experimental design. (B) The long-term survival of ICH mice with control or MCD diet after ICH. (C) NAFLD mice had more serious neurological function loss evaluated by mNSS test, corner turning test, and foot-fault test at days $7,14,21$, and 28 after $\mathrm{ICH}$. $n=10 \mathrm{mice}$ per group. Data are presented as mean $\pm \mathrm{SD}$. ${ }^{\star} P<0.05,{ }^{\star \star} P<0.01$

neurological deficits and brain water content at days 1 and 3 after $\mathrm{ICH}$ induction, as compared to control mice (Figures 1B-E), suggesting that NAFLD aggravates neurological dysfunction and brain injury in the acute stage of ICH.

We also evaluated the effects of NAFLD on the outcome of $\mathrm{ICH}$ at later time points. The results of mNSS, corner test, and foot fault test show that the detrimental effects of NAFLD on ICH persisted until at least 28 days after ICH induced by collagenase injection (Figures 2A,C). Furthermore, mice fed with NAFLD also have decreased survival index (Figure 2B). These results suggest that NAFLD could impair the outcome after ICH.

\section{NAFLD Augments Brain Inflammation Characterized by Increased Myeloid Cell Infiltration and MMP9 Production in the Brain After ICH}

Inflammation is a key factor causing secondary brain injury after ICH (Wang, 2010). We thus determined whether NAFLD has an impact on brain inflammation after ICH. Using flow cytometry, we examined the counts of infiltrating leukocytes as well as brain resident microglia at days 3 after ICH. The results showed that the counts of brain-infiltrating neutrophils $\left(\mathrm{CD} 45^{\text {high }} \mathrm{CD} 11 \mathrm{~b}^{+} \mathrm{Ly}_{6 \mathrm{G}}{ }^{+}\right)$and monocytes $\left(\mathrm{CD} 45^{\text {high }} \mathrm{CD} 11 \mathrm{~b}^{+}\right.$Ly6G $\mathrm{G}^{-}$Ly6 $\mathrm{C}^{\text {high }}$ ) were significantly increased in NAFLD mice compared to control mice at day 3 after ICH, but microglia and lymphocytes including $\mathrm{T}$ cells and $\mathrm{B}$ cells were not significantly different between these two groups (Figures 3A-C). Besides, RT-PCR data show that NAFLD also increased the mRNA levels of IL-1 $\beta$, CCL2, CXCL2, and MMP9 in the ipsilateral hemisphere after ICH, most of these genes are related to myeloid cell infiltration and function (Figure 3D).

Myeloid cells are the primary leukocyte subset to infiltrate the ICH brain and boost local inflammation by the production of pro-inflammatory factors. Among these factors, MMP9 is a pivotal contributor to $\mathrm{BBB}$ disruption. We found that the MFI (Mean Fluorescence Intensity) values of MMP9 in the brain-infiltrating neutrophils and monocytes were significantly increased in ICH mice receiving the MCD diet (Figure 4A). Besides, the extravasation of Evans blue dye was also aggravated in $\mathrm{ICH}$ mice receiving the MCD diet (Figure 4B). Together, these results indicate that NAFLD facilitates brain infiltration of myeloid cells and local inflammation, which may contribute to exacerbated $\mathrm{ICH}$ injury.

\section{NAFLD Mobilizes Neutrophils and Monocytes in the Periphery Before ICH}

To investigate the influence of NAFLD on immune responses, we compared the counts of immune cell subsets in spleens, 
A

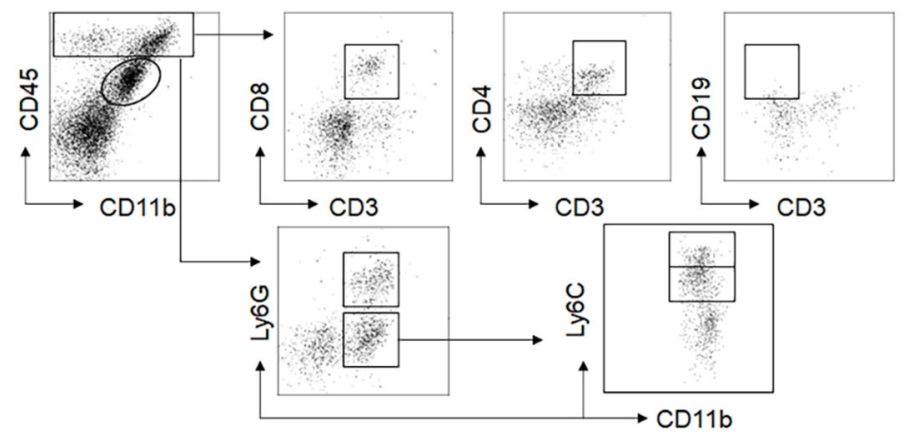

C

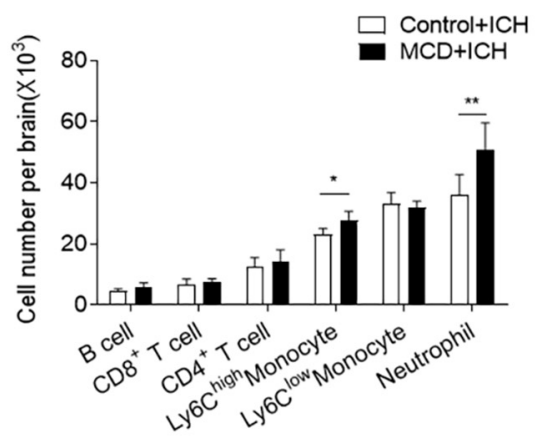

D

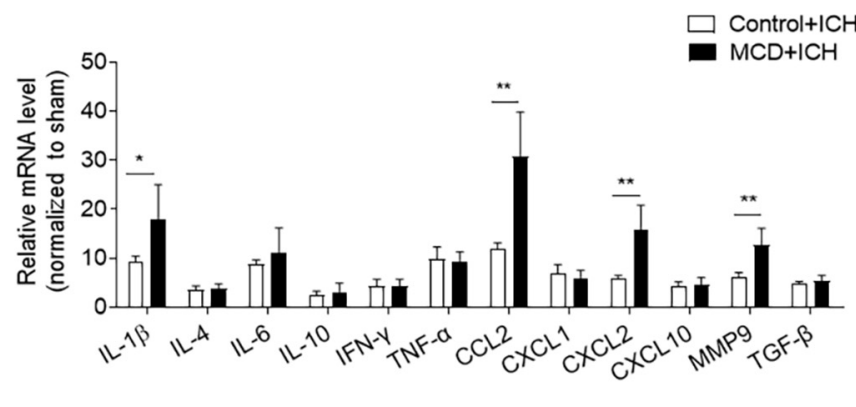

FIGURE 3 | NAFLD augments leukocyte infiltration and local inflammation in the brain after ICH. On day 3 after ICH induction by collagenase injection in NAFLD mice or control mice, the counts of immune cell subsets in the brain were measured by FACS on day 3 after ICH. (A) The gating strategy for microglia

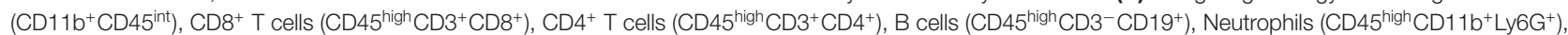
proinflammatory monocytes (CD45 high $\mathrm{CD} 11 \mathrm{~b}^{+}$Ly6G- Ly6C high) cells. (B,C) Bar graph illustrates the counts of indicated cell subsets in brains from ICH mice. $n=6$ per group. (D) The bar graph shows the expression of cytokines and chemokines in the ipsilateral hemispheres including IL-1 $\beta$, IL-4, IL-6, IL-10, IFN- $\gamma$, TNF- $\alpha$, CCL2, CXCL1, CXCL2, CXCL10, MMP9, and TGF- $\beta, n=6$ mice per group. Data are presented as mean \pm SD. ${ }^{\star} P<0.05,{ }^{\star \star} P<0.01$.

circulating blood, and brains of NAFLD mice and control mice before ICH. Flow cytometric analysis revealed that the number of neutrophils $\left(\mathrm{CD} 11 \mathrm{~b}^{+} \mathrm{Ly} 6 \mathrm{G}^{-} \mathrm{Ly} 6 \mathrm{C}^{\text {high }}\right)$ was significantly increased in the circulating blood and spleen from the mice with the MCD diet as compared to the control diet (Figures 5A,B). Pro-inflammatory monocytes $\left(\mathrm{CD} 11 \mathrm{~b}^{+}\right.$Ly6 $\left.\mathrm{C}^{\text {high }}\right)$ were also obviously increased in the blood (Figure 5A). In contrast, other immune cells including the counts of $\mathrm{CD}^{+} \mathrm{T}$ cells $\left(\mathrm{CD}^{+}{ }^{+} \mathrm{CD} 4^{+}\right), \mathrm{CD}^{+} \mathrm{T}$ cells $\left(\mathrm{CD}^{+} \mathrm{CD}^{+}\right)$, and $\mathrm{B}$ cells $\left(\mathrm{CD}^{-} \mathrm{CD} 19^{+}\right)$were not significantly altered in the spleen or circulating blood (Figures $\mathbf{5 A}, \mathbf{B}$ ). It is noteworthy that NAFLD did not have an impact on leukocyte infiltration or the number of microglia in the brain before ICH (Figure 5C). Our results indicate that NAFLD can mobilize neutrophils and monocytes in the periphery before $\mathrm{ICH}$, which may enhance the mobilization of these cells into the brain after ICH.

\section{Depletion of Myeloid Cells Alleviated NAFLD-Induced Exacerbation of Hemorrhagic Brain Injury}

Based on our previous data, we focused on studying the role of myeloid cells in the detrimental effects of NAFLD on ICH. We applied an anti-Gr-1 mAb (RB6-8C5) to deplete Gr-1 ${ }^{+}$ myeloid cells, including neutrophils and monocytes that are known participants in brain inflammation and BBB disruption (Hammond et al., 2014). Drug administration and experimental design were shown in Figure 6A. The efficacy of $\mathrm{Gr}-1^{+}$depletion was about 90\% (Supplementary Figure S2). We found the aggravation of hemorrhagic severity by NAFLD was much alleviated in the condition of $\mathrm{Gr}-1^{+}$cell depletion, displaying decreased brain water content and neurological deficit scores (Figures 6B,C). These results demonstrated that neutrophils and monocytes are involved in the detrimental effects of NAFLD on hemorrhagic brain injury.

\section{DISCUSSION}

This study provides the first evidence that NAFLD exacerbates hemorrhagic brain injury in two mouse models of ICH. As documented here, NAFLD not only aggravates neurological deficits and brain edema in the acute stage of ICH but also leads to persistent neurological dysfunction and reduced survival index. NAFLD augments leukocyte infiltration and the production of proinflammatory factors in the $\mathrm{ICH}$ brain. Importantly, we found that NAFLD mobilizes peripheral neutrophils and monocytes before ICH induction, suggesting that NAFLD induces mobilization of the peripheral immune 
A

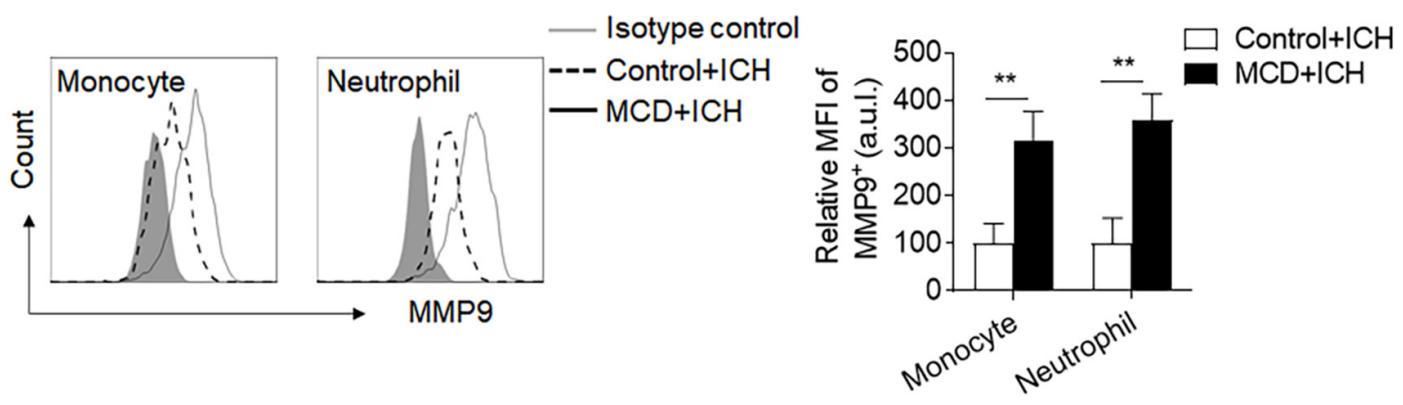

B
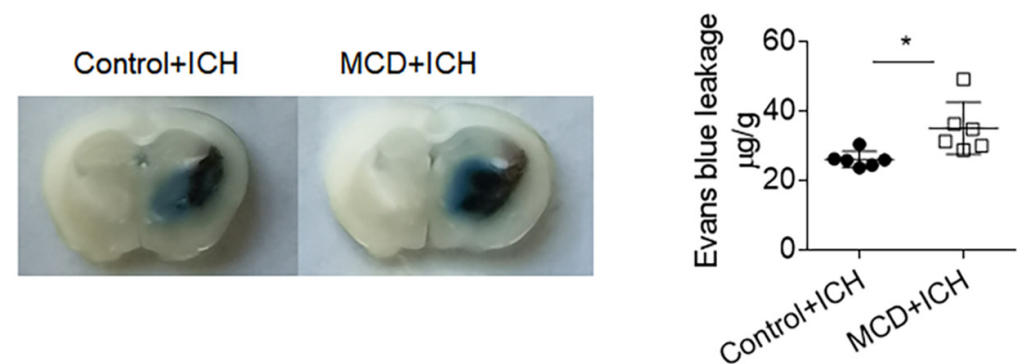

FIGURE 4 | NAFLD augmented MMP9 production and blood-brain barrier (BBB) disruption after ICH. ICH was induced by collagenase injection in NAFLD mice and control mice. MMP9 expressed by myeloid cells in mice brains was assessed at day 3 after ICH induction. (A) The mean fluorescence intensity of MMP9 in neutrophils and monocytes was quantified by flow cytometry. $n=6$ per group. (B) Histological images show that NAFLD increased Evans blue dye leakage at day 3 after ICH. $n=6$ mice per group. Data are presented as mean \pm SD. ${ }^{*} P<0.05,{ }^{\star \star} P<0.01$.

A

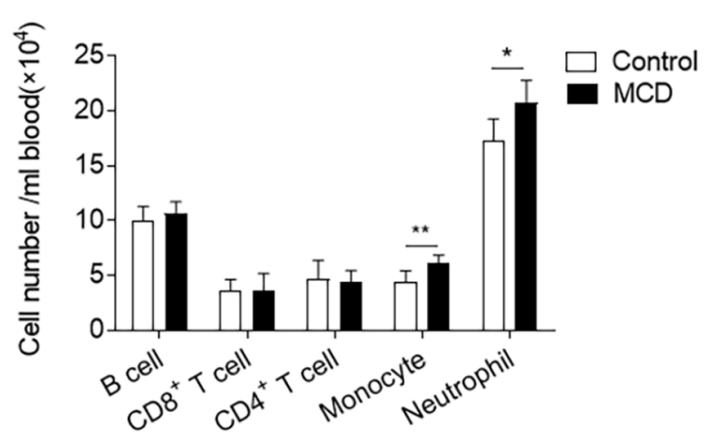

C

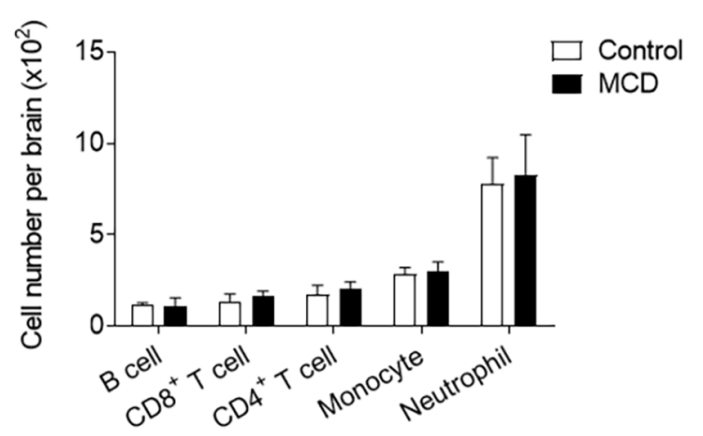

B
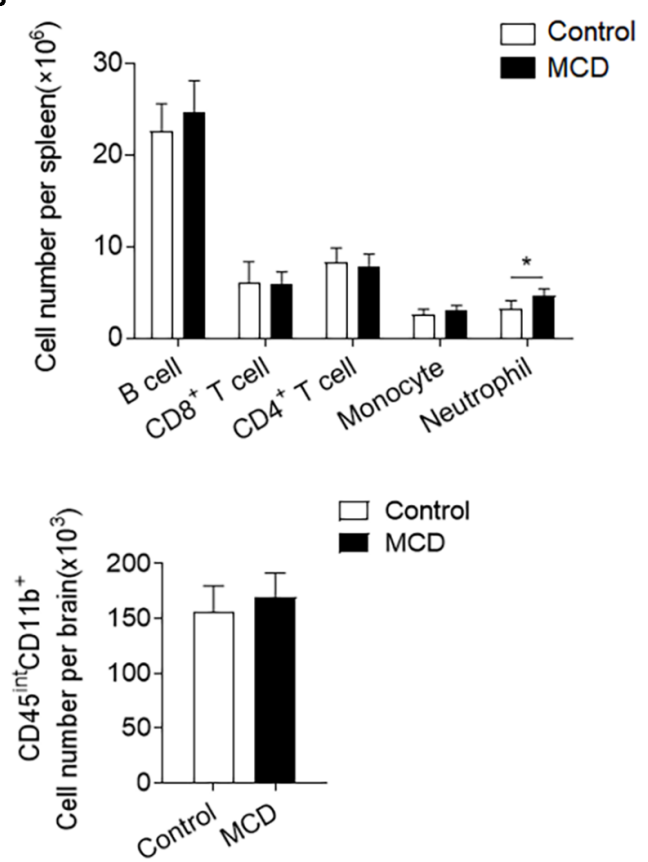

FIGURE 5 | NAFLD enhanced the mobilization of myeloid cells in the periphery. Immune cells were isolated from circulating blood, spleen, and brain tissues of NAFLD mice or control mice. (A,B) Summarized results of the counts of neutrophil (CD11 b+ ${ }^{+}$Ly6G $\left.{ }^{+}\right)$, Ly6C high monocytes (CD11 b ${ }^{+}$Ly6G $^{-}$Ly6C $\left.^{\text {high }}\right)$, CD4 ${ }^{+}$T cells $\left(\mathrm{CD}^{+} \mathrm{CD}^{+}\right), \mathrm{CD} 8^{+} \mathrm{T}$ cells $\left(\mathrm{CD} 3^{+} \mathrm{CD} 8^{+}\right)$and $\mathrm{B}$ cells $\left(\mathrm{CD} 3^{-} \mathrm{CD} 19^{+}\right)$in blood $(\mathbf{A})$ and spleen (B) of NAFLD mice and control mice. $n=6$ per group. (C) Summarized results of the counts of neutrophil $\left(\mathrm{CD} 45^{\text {high }} \mathrm{CD} 11 \mathrm{~b}^{+} \mathrm{Ly}_{6 \mathrm{G}}{ }^{+}\right.$), pro-inflammatory monocytes (CD45 $\left.{ }^{\text {high }} \mathrm{CD} 11 \mathrm{~b}^{+} \mathrm{Ly}_{6 \mathrm{G}}{ }^{-} \mathrm{Ly}_{6} \mathrm{C}^{\text {high }}\right), \mathrm{CD}^{+}{ }^{+} \mathrm{T}$ cells $\left(\mathrm{CD} 45^{\text {high }} \mathrm{CD} 3^{+} \mathrm{CD} 4^{+}\right)$, $\mathrm{CD}^{+} \mathrm{T}$ cells $\left(\mathrm{CD} 45^{\text {high }} \mathrm{CD}^{+} \mathrm{CD}^{+}\right)$, B cells $\left(\mathrm{CD} 45^{\text {high }} \mathrm{CD} 3^{-} \mathrm{CD} 19^{+}\right)$and microglia (CD45 int $\left.C D 11 \mathrm{~b}^{+}\right)$in brain of NAFLD mice and control mice. $n=6$ per group. Data are presented as mean \pm SD. ${ }^{\star} P<0.05,{ }^{\star \star} P<0.01$. 
A

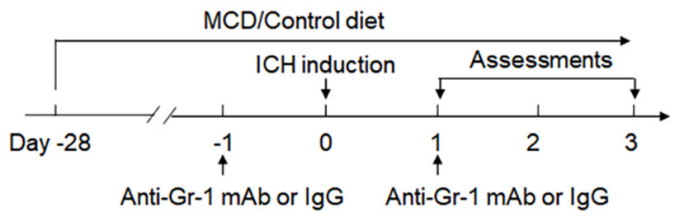

B

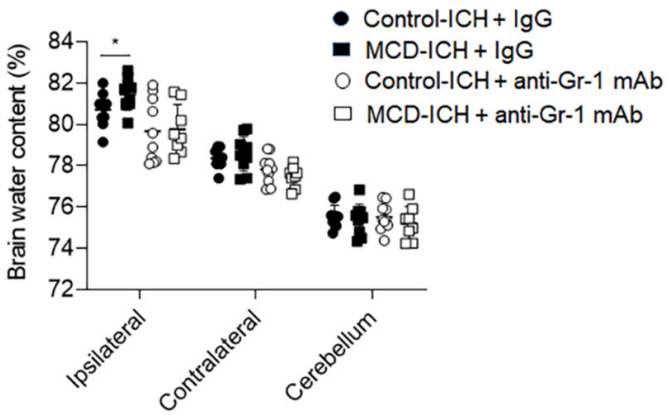

C

- Control-ICH+IgG a MCD-ICH + IgG O Control-ICH + anti-Gr-1 mAb $\square \mathrm{MCD}-\mathrm{ICH}+$ anti-Gr-1 mAb
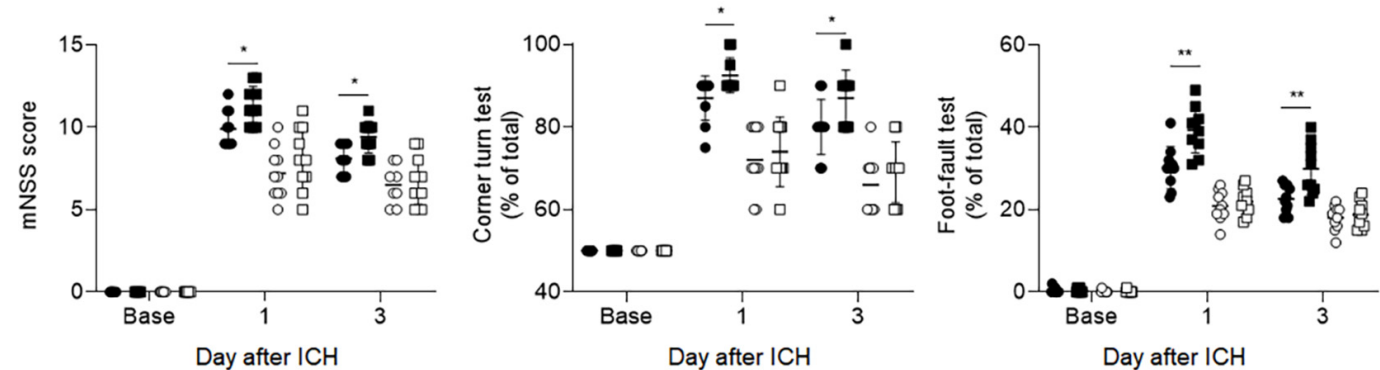

FIGURE 6 | The detrimental effect of NAFLD was abolished in ICH mice depleted of myeloid cells. (A) Flow chart illustrates drug administration and experimental design. NAFLD or control mice were given anti-Gr-1 mAb or IgG 1 day before and 1 day after $I C H$. Before $I C H$ and at days 1 and 3 after ICH, neurological deficits and brain water content were evaluated. (B) Results of the brain water content of mice receiving indicated treatments at day 3 after ICH. $n=10$ per group. (C) Statistical data of neurological deficits assessment including mNSS test, corner-turning test, and foot-fault test score in indicated groups of mice. $n=10$ per group. Data are presented as mean $\pm \mathrm{SD}$. ${ }^{\star} P<0.05,{ }^{\star \star} P<0.01$.

system. Also, results from antibody-mediated depletion of neutrophils and monocytes revealed the contribution of these cells to the detrimental effects of NAFLD on ICH.

Emerging evidence has demonstrated that brain inflammation exacerbates secondary brain injury and brain edema development after ICH (Wang, 2010). Neutrophils and monocytes are among early responders to ICH and migrate to the injury site at a very early stage of $\mathrm{ICH}$, leading to augmented local inflammation by producing a burst of pro-inflammatory factors. As previously documented, neutrophils and monocytes were observed in and around the hematoma as early as $4-5 \mathrm{~h}$ and peaked at 3 days after ICH onset (Wang and Tsirka, 2005; Wang and Dore, 2007). Reportedly, depletion of neutrophils in ICH animals reduced blood-brain barrier (BBB) breakdown, inflammation, and MMP-9 expression (Moxon-Emre and Schlichter, 2011). Consistent with these previous findings, we found that NAFLD increased MMP-9 production by braininfiltrating neutrophils and monocytes that may contribute to the $\mathrm{BBB}$ disruption and perihematomal edema. These results demonstrate that NAFLD augments brain inflammation after ICH, and highlight the involvement of neutrophils and monocytes in such processes.

NAFLD patients were found to have a persistent increase of circulating neutrophils and monocytes (Zhang et al., 2018; Khoury et al., 2019). As an extension of these previous findings, we found that mobilization of neutrophils and monocytes in the periphery contributes to the aggravation of hemorrhagic injury in
NAFLD mice. These results support the notion that neutrophils and monocytes are involved in the detrimental effects of NAFLD in ICH injury. It's also noteworthy that the number and activity of microglia were not affected by NAFLD, suggesting that NAFLD-induced mobilization of immune responses likely only occurs in the peripheral compartment rather than the brain, at least in our experimental setting. Together, our findings provide novel evidence regarding NAFLD-induced mobilization of neutrophils and monocytes, although the exact operating mechanisms through which NAFLD mobilizes neutrophils and monocytes and the subsets of these myeloid cells (i.e., neutrophils or monocytes) that is particularly involved in the pathological mechanisms of NAFLD to exacerbate ICH injury requires future investigation.

Changes in lifestyle and resultant over-nutrition are common causes of NAFLD (Farrell et al., 2013). More than 25\% of NAFLD patients develop potential nonalcoholic steatohepatitis (NASH) characterized by lipid accumulation, liver cell injury, and inflammation (Singh et al., 2015). In our study, we fed mice with an MCD diet for 4 weeks to induce NAFLD and hyperlipidemia. This is a well-established model to mimic human NAFLD features including hyperlipidemia, steatosis, inflammation, and fibrosis (Yu et al., 2010). Notably, we found unaltered coagulation time in NAFLD mice receiving MCD diet, suggesting that $\mathrm{MCD}$ diet-induced liver injury may not influence the coagulation or clotting processes, at least in the current experimental setting. Also, we are aware that the exacerbation 
of ICH injury in NAFLD animals cannot be entirely attributed to NAFLD-induced mobilization of peripheral neutrophils and monocytes. This is because NAFLD can also elicit other pathological events such as cerebrovascular alterations that may contribute to the predisposition of ICH injury. In this regard, these possibilities warrant future studies. Also, only male mice were used in this study. Future studies should also include female mice to clarify if there are any effects of sex differences in NAFLD-induced aggravation of ICH injury.

In summary, our findings suggest that the mobilization of neutrophils and monocytes in NAFLD mice contributes to aggravated hemorrhagic brain injury. We, therefore, infer that the prevention and control of NAFLD may reduce the detrimental consequences of $\mathrm{ICH}$.

\section{CONCLUSION}

We demonstrate that NAFLD worsens neurological deficits and brain injury after $\mathrm{ICH}$, accompanied by augmented brain infiltration of neutrophils and monocytes and the production of pro-inflammatory factors. Our data suggest that the prevention and control of NAFLD would reduce the detrimental consequences of ICH.

\section{DATA AVAILABILITY STATEMENT}

All datasets generated for this study are included in the article/Supplementary Material.

\section{REFERENCES}

Aronowski, J., and Zhao, X. (2011). Molecular pathophysiology of cerebral hemorrhage: secondary brain injury. Stroke 42, 1781-1786. doi: 10.1161/strokeaha.110.596718

Clarkson, A. N., Huang, B. S., Macisaac, S. E., Mody, I., and Carmichael, S. T. (2010). Reducing excessive GABA-mediated tonic inhibition promotes functional recovery after stroke. Nature 468, 305-309. doi: 10.1038/nature09511

Condamine, T., Kumar, V., Ramachandran, I. R., Youn, J. I., Celis, E., Finnberg, N., et al. (2014). ER stress regulates myeloid-derived suppressor cell fate through TRAIL-R-mediated apoptosis. J. Clin. Invest. 124, 2626-2639. doi: 10.1172/jci74056

Farrell, G. C., van Rooyen, D., Gan, L., and Chitturi, S. (2012). NASH is an inflammatory disorder: pathogenic, prognostic and therapeutic implications. Gut Liver 6, 149-171. doi: 10.5009/gnl.2012.6.2.149

Farrell, G. C., Wong, V. W., and Chitturi, S. (2013). NAFLD in Asia-as common and important as in the West. Nat. Rev. Gastroenterol. Hepatol. 10, 307-318. doi: 10.1038/nrgastro.2013.34

Fiorucci, S., Biagioli, M., and Distrutti, E. (2018). Future trends in the treatment of non-alcoholic steatohepatitis. Pharmacol. Res. 134, 289-298. doi: 10.1016/j. phrs.2018.07.014

Gao, B., and Tsukamoto, H. (2016). Inflammation in alcoholic and nonalcoholic fatty liver disease: friend or foe? Gastroenterology 150, 1704-1709. doi: 10.1053/j.gastro.2016.01.025

Gluchowski, N. L., Becuwe, M., Walther, T. C., and Farese, R. V. Jr. (2017). Lipid droplets and liver disease: from basic biology to clinical implications. Nat. Rev. Gastroenterol. Hepatol. 14, 343-355. doi: 10.1038/nrgastro. 2017.32

Hammond, M. D., Taylor, R. A., Mullen, M. T., Ai, Y., Aguila, H. L., Mack, M., et al. (2014). CCR2 ${ }^{+}$Ly6C $^{\text {hi }}$ inflammatory monocyte recruitment exacerbates

\section{ETHICS STATEMENT}

All animal experiments were reviewed and approved by the Institutional Animal Care and Use Committees of Tianjin Medical University General Hospital (Tianjin, China). This study was conducted following the National Institutes of Health Guide for the Care and Use of Laboratory Animals in China.

\section{AUTHOR CONTRIBUTIONS}

ML, QL, and HM formulated the concept and designed the study. $\mathrm{XL}$ and XC performed the studies. XL and XW analyzed the data and interpreted the results. ML, QL, and XL wrote the article.

\section{FUNDING}

This study was supported by Key Projects of Natural Science Foundation of Tianjin (18JCZDJC97600) and National Natural Science Foundation of China (81701176).

\section{ACKNOWLEDGMENTS}

We thank Kristofer Wood for editorial assistance.

\section{SUPPLEMENTARY MATERIAL}

The Supplementary Material for this article can be found online at: https://www.frontiersin.org/articles/10.3389/fncel. 2020.00154/full\#supplementary-material.

acute disability following intracerebral hemorrhage. J. Neurosci. 34, 3901-3909. doi: 10.1523/JNEUROSCI.4070-13.2014

Han, R., Luo, J., Shi, Y., Yao, Y., and Hao, J. (2017). PD-L1 (programmed death ligand 1) protects against experimental intracerebral hemorrhage-induced brain injury. Stroke 48, 2255-2262. doi: 10.1161/strokeaha.117.016705

Haukeland, J. W., Damås, J. K., Konopski, Z., Løberg, E. M., Haaland, T., Goverud, I., et al. (2006). Systemic inflammation in nonalcoholic fatty liver disease is characterized by elevated levels of CCL2. J. Hepatol. 44, 1167-1174. doi: 10.1016/j.jhep.2006.02.011

Jin, W. N., Yang, X., Li, Z., Li, M., Shi, S. X., Wood, K., et al. (2016). Noninvasive tracking of $\mathrm{CD}^{+} \mathrm{T}$ cells with a paramagnetic and fluorescent nanoparticle in brain ischemia. J. Cereb. Blood Flow Metab. 36, 1464-1476. doi: $10.1177 / 0271678 \times 15611137$

Khoury, T., Mari, A., Nseir, W., Kadah, A., Sbeit, W., and Mahamid, M. (2019). Neutrophil-to-lymphocyte ratio is independently associated with inflammatory activity and fibrosis grade in nonalcoholic fatty liver disease. Eur. J. Gastroenterol. Hepatol. 31, 1110-1115. doi: 10.1097/meg.0000000000001393

Kim, H. L., Chung, G. E., Park, I. Y., Choi, J. M., Hwang, S. M., Lee, J. H., et al. (2011). Elevated peripheral blood monocyte fraction in nonalcoholic fatty liver disease. Tohoku J. Exp. Med. 223, 227-233. doi: 10.1620/tjem.223.227

Klebe, D., Flores, J. J., McBride, D. W., Krafft, P. R., Rolland, W. B., Lekic, T., et al. (2017). Dabigatran ameliorates post-haemorrhagic hydrocephalus development after germinal matrix haemorrhage in neonatal rat pups. J. Cereb. Blood Flow Metab. 37, 3135-3149. doi: 10.1177/0271678x16684355

Larter, C. Z., and Yeh, M. M. (2008). Animal models of NASH: getting both pathology and metabolic context right. J. Gastroenterol. Hepatol. 23, 1635-1648. doi: 10.1111/j.1440-1746.2008.05543.x

Li, M., Li, Z., Ren, H., Jin, W. N., Wood, K., Liu, Q., et al. (2017a). Colony stimulating factor 1 receptor inhibition eliminates microglia and attenuates brain injury after intracerebral hemorrhage. J. Cereb. Blood Flow Metab. 37, 2383-2895. doi: 10.1177/0271678x16666551 
Li, M., Ren, H., Sheth, K. N., Shi, F. D., and Liu, Q. (2017b). A TSPO ligand attenuates brain injury after intracerebral hemorrhage. FASEB J. 31, 3278-3287. doi: 10.1096/fj.201601377rr

Liu, Q., Sanai, N., Jin, W. N., La Cava, A., Van Kaer, L., and Shi, F. D. (2016). Neural stem cells sustain natural killer cells that dictate recovery from brain inflammation. Nat. Neurosci. 19, 243-252. doi: 10.1038/ nn. 4211

Moxon-Emre, I., and Schlichter, L. C. (2011). Neutrophil depletion reduces bloodbrain barrier breakdown, axon injury, and inflammation after intracerebral hemorrhage. J. Neuropathol. Exp. Neurol. 70, 218-235. doi: 10.1097/nen. 0b013e31820d94a5

Mracsko, E., Javidi, E., Na, S. Y., Kahn, A., Liesz, A., and Veltkamp, R. (2014). Leukocyte invasion of the brain after experimental intracerebral hemorrhage in mice. Stroke 45, 2107-2114. doi: 10.1161/strokeaha.114. 005801

Ren, H., Kong, Y., Liu, Z., Zang, D., Yang, X., Wood, K., et al. (2018). Selective NLRP3 (pyrin domain-containing protein 3) inflammasome inhibitor reduces brain injury after intracerebral hemorrhage. Stroke 49, 184-192. doi: 10.1161/strokeaha.117.018904

Sheth, K. N., and Rosand, J. (2014). Targeting the immune system in intracerebral hemorrhage. JAMA Neurol. 71, 1083-1084. doi: 10.1001/jamaneurol.2014.1653

Singh, S., Allen, A. M., Wang, Z., Prokop, L. J., Murad, M. H., and Loomba, R. (2015). Fibrosis progression in nonalcoholic fatty liver vs nonalcoholic steatohepatitis: a systematic review and meta-analysis of paired-biopsy studies. Clin. Gastroenterol. Hepatol. 13, 643.e1-654.e9; quiz e39-e40. doi: 10.1016/j. cgh.2015.02.012

Sun, N., Shen, Y., Han, W., Shi, K., Wood, K., Fu, Y., et al. (2016). Selective sphingosine-1-phosphate receptor 1 modulation attenuates experimental intracerebral hemorrhage. Stroke 47, 1899-1906. doi: 10.1161/strokeaha.115. 012236

Tsochatzis, E. A., and Newsome, P. N. (2018). Non-alcoholic fatty liver disease and the interface between primary and secondary care. Lancet Gastroenterol. Hepatol. 3, 509-517. doi: 10.1016/s2468-1253(18)30077-3

Wang, J. (2010). Preclinical and clinical research on inflammation after intracerebral hemorrhage. Prog. Neurobiol. 92, 463-477. doi: 10.1016/j. pneurobio.2010.08.001

Wang, J., and Dore, S. (2007). Heme oxygenase-1 exacerbates early brain injury after intracerebral haemorrhage. Brain 130, 1643-1652. doi: 10.1093/brain/awm095

Wang, H., Hong, L. J., Huang, J. Y., Jiang, Q., Tao, R. R., Tan, C., et al. (2015). P2RX7 sensitizes Mac-1/ICAM-1-dependent leukocyte-endothelial adhesion and promotes neurovascular injury during septic encephalopathy. Cell Res. 25, 674-690. doi: $10.1038 / \mathrm{cr} .2015 .61$
Wang, J., and Tsirka, S. E. (2005). Neuroprotection by inhibition of matrix metalloproteinases in a mouse model of intracerebral haemorrhage. Brain 128, 1622-1633. doi: 10.1093/brain/awh489

Yang, C. S., Guo, A., Li, Y., Shi, K., Shi, F. D., and Li, M. (2019). Dl-3-nbutylphthalide reduces neurovascular inflammation and ischemic brain injury in mice. Aging Dis. 10, 964-976. doi: 10.14336/ad.2019.0608

Younossi, Z., Anstee, Q. M., Marietti, M., Hardy, T., Henry, L., Eslam, M., et al. (2018). Global burden of NAFLD and NASH: trends, predictions, risk factors and prevention. Nat. Rev. Gastroenterol. Hepatol. 15, 11-20. doi: 10.1038/nrgastro.2017.109

Yu, J., Chu, E. S., Wang, R., Wang, S., Wu, C. W., Wong, V. W., et al. (2010). Heme oxygenase-1 protects against steatohepatitis in both cultured hepatocytes and mice. Gastroenterology 138, 694-704, 704.e1. doi: 10.1053/j.gastro.2009.09.058

Zhang, J., Chen, W., Fang, L., Li, Q., Zhang, X., Zhang, H., et al. (2018) Increased intermediate monocyte fraction in peripheral blood is associated with nonalcoholic fatty liver disease. Wien. Klin. Wochenschr. 130, 390-397. doi: 10.1007/s00508-018-1348-6

Zhang, Y., Han, B., He, Y., Li, D., Ma, X., Liu, Q., et al. (2017). MicroRNA132 attenuates neurobehavioral and neuropathological changes associated with intracerebral hemorrhage in mice. Neurochem. Int. 107, 182-190. doi: 10.1016/j.neuint.2016.11.011

Zhang, X., Shen, J., Man, K., Chu, E. S., Yau, T. O., Sung, J. C., et al. (2014). CXCL10 plays a key role as an inflammatory mediator and a non-invasive biomarker of non-alcoholic steatohepatitis. J. Hepatol. 61, 1365-1375. doi: 10.1016/j.jhep.2014.07.006

Zhang, Z., Zhang, Z., Lu, H., Yang, Q., Wu, H., and Wang, J. (2017). Microglial polarization and inflammatory mediators after intracerebral hemorrhage. $\mathrm{Mol}$. Neurobiol. 54, 1874-1886. doi: 10.1007/s12035-016-9785-6

Zhou, Y., Wang, Y., Wang, J., Anne Stetler, R., and Yang, Q. W. (2014). Inflammation in intracerebral hemorrhage: from mechanisms to clinical translation. Prog. Neurobiol. 115, 25-44. doi: 10.1016/j.pneurobio.2013.11.003

Conflict of Interest: The authors declare that the research was conducted in the absence of any commercial or financial relationships that could be construed as a potential conflict of interest.

Copyright (c) 2020 Li, Cheng, Wang, Liu, Ma and Li. This is an open-access article distributed under the terms of the Creative Commons Attribution License (CC BY). The use, distribution or reproduction in other forums is permitted, provided the original author(s) and the copyright owner(s) are credited and that the original publication in this journal is cited, in accordance with accepted academic practice. No use, distribution or reproduction is permitted which does not comply with these terms. 